\title{
Scope of Negation and Clause Structure in Japanese ${ }^{1}$
}

\author{
CHUNG-HYE HAN, DENNIS RYAN STOROSHENKO, YASUKO SAKURAI \\ Simon Fraser University
}

\section{Introduction}

Japanese has two ways of forming sentential negation: regular negation with -na inflected on the main verb, as in (1a), and wa-negation with the so-called topic marker -wa on the main verb followed by auxiliary suru inflected with -na, as in $(1 \mathrm{~b}) .^{2}$

(1) a. Donald-ga orenji subete-o tabe-na-katta.

Donald-NOM orange every-ACC eat-NEG-PST

'Donald did not eat every orange.'

b. Donald-ga orenji subete-o tabe-wa shi-na-katta.

Donald-NOM orange every-ACC eat-TOP do-NEG-PST

'Donald did not eat every orange.'

The purpose of this paper is to provide a unified syntactic analysis of the two types of negation based on data concerning the scope of negation and a quantified object NP (object QNP), extracted through psycholinguistic experimentation using a technique known as the Truth Value Judgment Task (TVJT) (Crain and Thornton, 1998). We will argue that the findings of our experiment support a view that the negation projection is placed low in the clause structure, within the lexical $v \mathrm{P}$ domain.

This paper is organized as follows. In section 1, we consider lexical and syntactic approaches to Japanese negation and conclude that the data calls for the syntactic approach with a negation projection (NegP) in the clause structure. We then present an argument that the scope interaction between negation and the object QNP makes a great test for the position of NegP in Japanese. We point out, however, that the data cannot be used as it is presented in the literature on this topic because the scope judgments reported there conflict with each other, making it impossible for us to make any coherent conclusions. This raises a question as to the validity of the methodology employed in extracting these scope judgments. In section 2, we present the TVJT experiment that we conducted in order to extract more reliable scope judgments. After a discussion of the particulars of our experiment, we present our findings followed by their implications for the syntax of negation in Japanese.

\footnotetext{
${ }^{1}$ We thank the audience at BLS 30 for helpful questions and comments. The work reported in this paper was supported by SSHRC \#410-2003-0544 to the first author.

${ }^{2}$ We gloss -wa as TOP 'topic marker' for lack of a better term, but stay neutral as to the discourse function of this marker.
} 


\section{Negation Projection}

\subsection{Motivating a Negation Projection}

In principle, negation - $n a$ can be incorporated into the clause structure in two alternative ways: lexically or structurally. Under the lexical approach, $-n a$ is a derivational morpheme, entering the syntactic derivation as a single syntactic unit along with the verb it is affixed onto. Under the structural approach, $-n a$ is an inflectional morpheme, projecting its own functional projection (NegP), and combining with the verb in morphology.

The form of the tense inflection on the verb occurring with -na appears to support the lexical approach. In Japanese, the tense inflection on the adjective is distinct from the verb. For example, while the past tense morpheme on the verb tabe 'eat' is -ta as in (2a), the one on the adjective oki 'big' is -katta as in (2b). It turns out that negated verbs pattern with adjectives, not with verbs, taking the adjective tense inflection, as in (2c).
a. Jiroo-ga piza-o tabe-ta.
Jiroo-NOM pizza-ACC eat-PST
'Jiroo ate pizza.'
b. Hon-wa oki-katta.
book-TOP big-PST
'The book was big.'
c. Jiroo-ga piza-o tabe-na-katta.
Jiroo-NOM pizza-ACC eat-NEG-PST
'Jiroo did not eat pizza.'

This patterning of tense inflection seems to suggest that -na is a derivational morpheme that derives an adjectival category. However, there are at least two facts that support the structural approach to -na. First, syntactically, negated verbs still behave like verbs. For example, transitive verbs usually assign an accusative case to their internal arguments as in (2a), while transitive adjectives usually assign a nominative case to them as in (3). The internal argument in negated verbs bears accusative case, hence patterning as a verb, not as an adjective, as in (2c).

\section{(3) Noriko-wa Shuya-ga suki desu. \\ Noriko-TOP Shuya-NOM fond of be \\ 'Noriko is fond of Shuya.'}

Further evidence supporting the structural approach comes from the fact that - na triggers weak island effects. While object extraction from an embedded clause across a negative matrix predicate is possible as in (4a), adjunct extraction in the same context is not possible (Hoji, 1985; Miyagawa, 2002). In (4b), only the reading in which the time of Shuya's thinking is questioned is available.
a. Nani-O ${ }_{i}$
Hanako-shika [Taroo-ga $t_{i}$ kat-ta to] what-ACC ${ }_{i}$ Hanako-only Taroo-NOM $t_{i}$ buy-PST COMP
omottei-na-i-no?
think-NEG-NPST-Q
'What does only Hanako think that Taroo bought?' 
b. Shuya-ga itsu Noriko-ga suupu-o non-da to Shuya-NOM when Noriko-NOM soup-ACC drink-PST COMP omo-ana-katta-no? think-NEG-PST-Q

'When didn't Shuya think Noriko drank soup?'

According to Rizzi (1990), an empty operator in [Spec,NegP] is responsible for weak island effects in negative sentences. The same type of weak island effect found in Japanese negative sentences thus provides evidence for an empty operator in [Spec,NegP], which in turn is evidence for positing a phrasal projection for negation.

\subsection{Placement of the Negation Projection}

We have so far established that Japanese negation projects a NegP. The question now is where in the clause structure NegP is projected, and whether there are two different positions for NegP, given that Japanese has two ways of forming sentential negation, regular and wa-negation, as was illustrated in (1). The fact that the two negations cannot be combined to form a double negation, as in (5), suggests that there is only one NegP position in Japanese clause structure.

$$
\begin{aligned}
& \text { * Donald-ga orenji subete-o tabe-na-wa shi-na-katta. } \\
& \text { Donald-NOM orange every-ACC eat-NEG-TOP do-NEG-PST } \\
& \text { 'Donald didn't not eat every orange.' }
\end{aligned}
$$

The issue of determining the exact position of NegP turns out to be a difficult one, even though there is a good diagnostic for it, namely scope interaction between negation and object QNP. Two independently motivated background facts about Japanese syntax make scope facts informative. First, scope for argument NPs is fixed before, and not at, LF in Japanese, as independently motivated by Scope Rigidity effects (Kuno, 1973; Kuroda, 1979; Hoji, 1985). That is, the scope of argument QNPs is determined by the surface c-command relationship, without recourse to QR or reconstruction. Thus, a sentence with canonical SOV word order containing quantifiers in both subject and object NPs only exhibits the reading in which the subject scopes over the object, as in (6).

(6) Dareka-ga ooku-no hitobito-o hihanshi-ta. someone-NOM many-GEN people-ACC criticize-PST

'Someone criticized many people.' ( $\sqrt{ }$ some $>$ many, $*$ many $>$ some $)$

Second, it is standardly assumed that the object NP in Japanese undergoes object raising to $[\mathrm{Spec}, v \mathrm{P}]$ to check accusative case, forming a multiple specifier with the external argument, the subject, as in (7) (Chomsky, 1995; Koizumi, 1995; Miyagawa, 2001). 
(7)

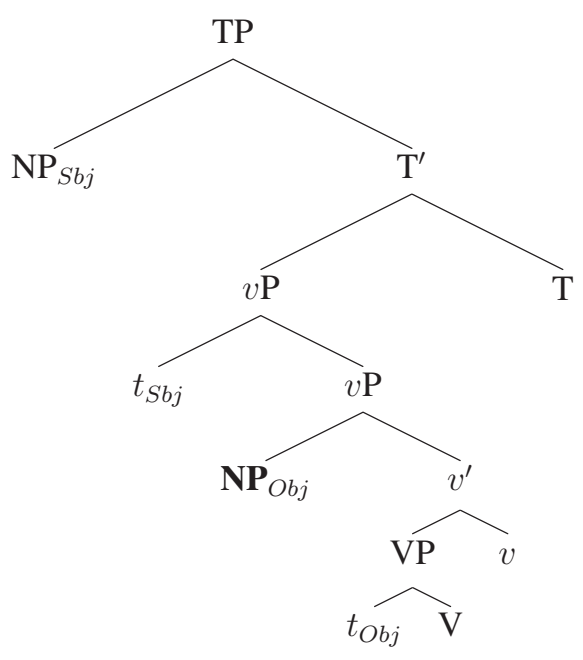

Positing multiple specifiers of $v$, one for the external argument and the other for the purposes of accusative case checking, captures the tight connection between the external argument and accusative case, as reflected in Burzio's Generalization. In passives, $v$ would be defective, neither having the ability to check accusative case nor host an external argument, hence lacking both of the specifiers.

Putting the two background facts together, a prediction emerges. Starting with the clause structure in (7), there are two possible positions for NegP in principle: NegP could be low in the clause structure within $v \mathrm{P}$ as in Hypothesis 1 in (8a), or it could be higher in the clause structure above $v \mathrm{P}$ as in Hypothesis 2 in (8b).

a. Hypothesis 1

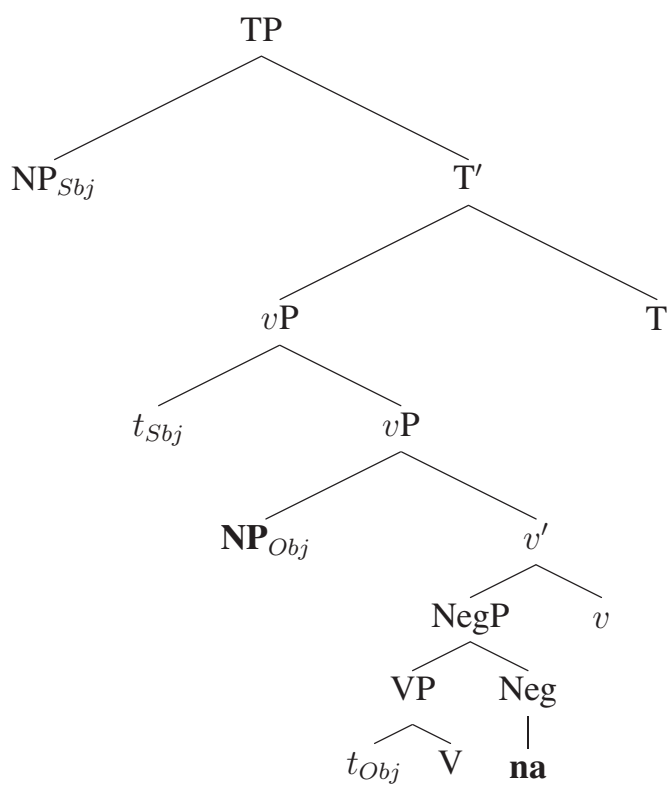

b. Hypothesis 2

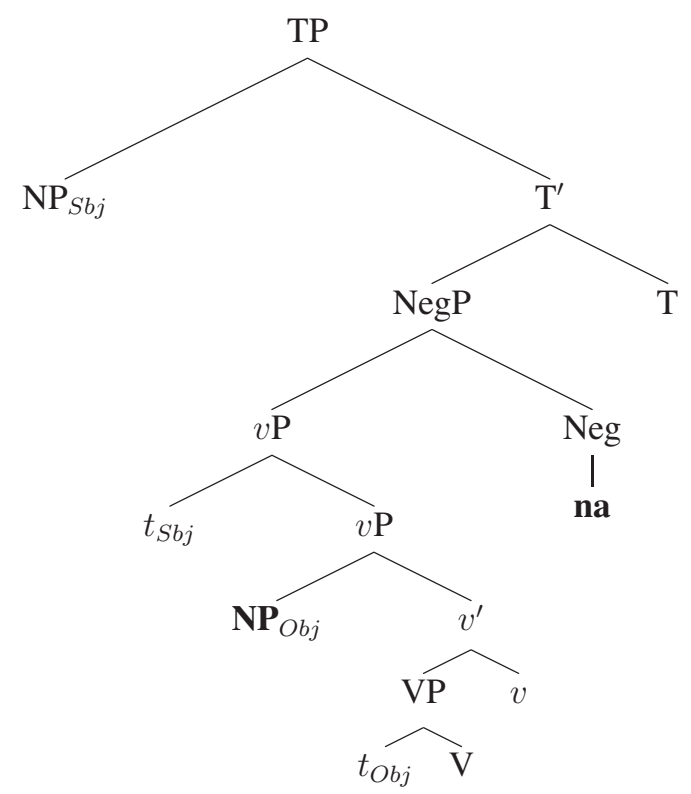

Further, given Scope Rigidity, in a negative sentence with an object QNP in its canonical position, the relative scope of negation and the object QNP will directly reflect their relative positions with respect to each other. This then means that the two hypotheses in (8) make different predictions: Hypothesis 1 predicts that object 
QNP scopes over negation, and Hypothesis 2 predicts that negation scopes over object QNP. The predictions are clear, but unfortunately, the claims made in the literature about this topic are not.

\subsection{Disagreement in the Literature}

When examining the relationship between sentential negation and an object QNP, there are ultimately three possible relations: $\mathrm{Neg}>\mathrm{Q}, \mathrm{Q}>\mathrm{Neg}$, or ambiguity in which, for a given sentence, either relation can hold. In examining the existing literature on the subject, all three of these positions can be found, with the further complication that opinions can vary depending upon the type of negation being used. Kuno (1980) enters this discussion with the claim that verbally-adjoined negation takes scope only over the immediately preceding verb, but amends this claim when examining a case with an object QNP:

(9) Pai-o zenbu tabe-rare-na-katta.

Pie-ACC all eat-can-NEG-PST

'I could not eat all the pie.'

For examples like (9), Kuno claims that the reading should primarily be Neg $>$ Q, despite his earlier claim that negation should take the narrowest possible scope. The alternative reading of $\mathrm{Q}>\mathrm{Neg}$ is given as a possible secondary reading. The notion that an object QNP should take scope over negation arises in Yatabe (1996), where it is claimed that all quantifiers in Japanese should take scope over negation. In this analysis, readings of $\mathrm{Neg}>\mathrm{Q}$ are not attained whatsoever.

The possibility of outright ambiguity emerges in Ota and Kato (1986), where examples such as (9) are given as equally Neg $>$ Q or Q $>$ Neg, without hedges such as referring to primary or secondary readings. In attempting to solve a separate syntactic puzzle, Miyagawa (2001) uses both subject and object QNP scope judgments as a source of evidence. Here, while the overall appraisal seems to agree with Kuno in that Neg $>$ Q seems to be the preferred reading when encountering an object QNP, Miyagawa notes that for some consultants, Q $>$ Neg was also possible. Thus, while Kuno and others seem to believe that regular negation sentences are subtly ambiguous, Miyagawa introduces the notion that they may only be so for some native speakers.

There is much less said in the literature on the subject of the relative scope between object QNP's and wa-negation. This being the case, what little there is to be found is noteworthy in that unlike the discussion of regular negation's scope, all discussions of $w a$-negation scope are consistent. There is general agreement with the stance taken in Ota and Kato (1986) that in wa-negation, negation should always scope over the object QNP.

\section{Experimental Investigation}

Given the conflicting claims in the literature on the scope judgments, we cannot use them as they are to make any conclusions as to which hypothesis is superior. What could be the source of the disagreement in the literature? One possibility might be the methodological problem in the elicitation of scope judgments. Some speakers may have difficulty in identifying a reading associated with a sentence without a sufficient discourse context. Another possibility might be that the disagreement actually reflects speakers' grammars. It is possible that different speakers have 
different grammars, resulting in the apparent disagreement in scope judgments. To address these issues, we designed a psycholinguistic experiment using the TVJT technique and extracted scope judgments that we think clearly reflect speakers' grammars.

\subsection{Methodology}

The TVJT involves two experimenters. One experimenter acts out short stories in front of the participant using toys. The other experimenter plays the role of a puppet (e.g., Mickey Mouse) who watches the scenario alongside the participant. At the end of the story, the puppet makes a statement about the story. The participant's task is to determine whether the puppet understood the story and say whether it told the truth or not.

To test how speakers interpret a sentence with negation and an object QNP, such as Donald didn't eat every orange, an experimenter enacts a story, using Donald Duck, three oranges, and three watermelons. Donald Duck is hungry and finds three oranges and three watermelons. He then eats all three watermelons, but eats only two oranges. In figure (10), the picture in the left is a shot of the beginning of the scenario, and the one in the right is a shot of the end of the scenario. After the enactment is over, the puppet is prompted to say what just happened. It then makes the statement in (11).

\section{(10) Context}
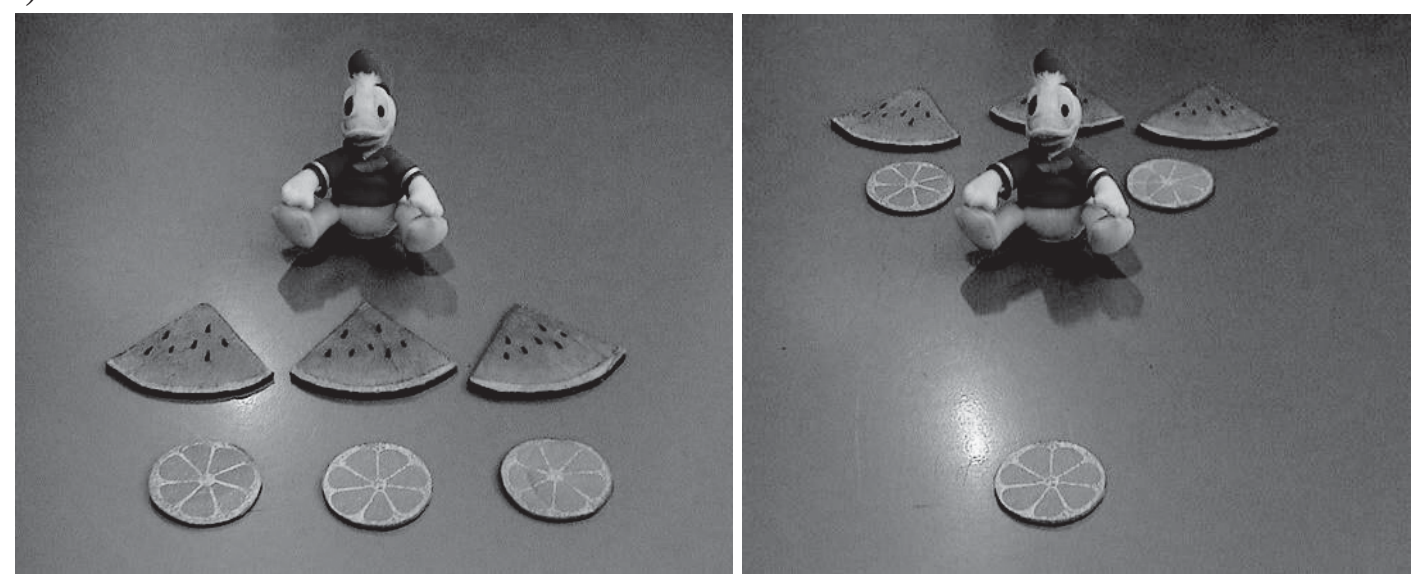

(11)

\section{Puppet statement: \\ "I know what happened. Donald didn't eat every orange. Am I right?"}

In this story, the reading in which negation scopes over the object QNP (Neg $>\forall$ ) yields true, and the reading in which the object QNP scopes over negation $(\forall>$ Neg) yields false. So if a participant accepts (11) in this context, then we can conclude that the grammar makes available the wide scope reading of negation. But if a participant rejects (11), we can conclude that it must be because the grammar does not generate $\mathrm{Neg}>\forall$ interpretation.

The TVJT provides rich discourse contexts in a simple method, with not much memory load on the participants. It has been shown to work in several languages, such as English and Kannada in Lidz and Musolino (2002) and Korean in Han et al. (2003), and to work with both adults and children as young as 4 years old (Crain 
and McKee, 1985; Crain and Thornton, 1998; Lidz and Musolino, 2002; Han et al., 2003).

The overall design of our experiment and the stories we used in the TVJT are closely modelled after the work reported in Han et al. (2003). We designed the experiment to test 2 factors with 2 levels each: negation type (regular neg vs. waneg) $\times$ scope $(\forall>$ Neg vs. Neg $>\forall)$. The experiment was thus divided into 4 different conditions, each condition testing for the $\forall>$ Neg or Neg $>\forall$ reading in sentences containing regular or $w a$-negation. For each condition, we tested 12 participants, a total of 48 participants for the entire experiment. They are 20 - to 30-year-old Japanese native speakers living in Vancouver at the time of the testing, who had spent no more than a combined span of 12 months in North America or any other English-speaking area. The design is summarized in table (12).

Experimental Design
\begin{tabular}{|l||l|l|}
\hline Scope & Plain negation & $w a$-negation \\
\hline \hline$\forall>$ Neg & $n=12$ & $n=12$ \\
\hline Neg $>\forall$ & $n=12$ & $n=12$ \\
\hline
\end{tabular}

Participants were tested individually in a small classroom. Each participant was first introduced to the task with 4 practice trials. They were then given 4 test trials and 4 filler trials in a pseudorandom order. In a brief debriefing session at the end of all the trials, they were asked to provide a justification for their answers. This allowed us to check whether the answers of the participants were based on the right reasons and not on irrelevant factors. All scenarios were pre-recorded and were shown to each participant on a portable computer screen. The use of pre-recorded scenarios allowed us to avoid any variation in the enactment of scenarios and to keep the prosody of the test statements constant. The test sentences given to the participants each contained an object QNP with either regular or wa-negation, as in (1), repeated here as (13). In the scenario that tests the Neg $>\forall$ reading, Donald eats two oranges out of three, and in the scenario that tests the $\forall>$ Neg reading, Donald eats none of the oranges.
a. Donald-ga orenji subete-o tabe-na-katta. Donald-NOM orange every-ACC eat-NEG-PST
'Donald did not eat every orange.'
b. Donald-ga orenji subete-o tabe-wa shi-na-katta. Donald-NOM orange every-ACC eat-TOP do-NEG-PST
'Donald did not eat every orange.'

The purpose of the filler trials was to separately test the participants' comprehension of negation and of QNPs, and to prevent any priming effects. All participants were given two filler sentences containing a subject QNP, as in (14a), and the participants in regular negation conditions were given two filler sentences with $w a$-negation, as in (14b), while those in $w a$-negation conditions were given two filler sentences with regular negation, as in (14c). To introduce variation in the answers, we set up the filler scenarios such that two of the fillers should be true and the other two should be false. 
(14)

a. Otokonohito subete-ga iwa-o nage-ta.
man every-NOM rock-ACC throw-PST

'Every man threw a rock.'

b. Zou-ga kirikabu-ni nobori-wa shi-na-katta. elephant-NOM tree-trunk-onto climb-TOP do-NEG-PST

'The elephant did not climb onto the tree trunk.'

c. Zou-ga kirikabu-ni nobor-ana-katta.

elephant-NOM tree-trunk-onto climb-NEG-PST

'The elephant did not climb onto the tree trunk.'

\subsection{Results}

To obtain quantified results, whenever a participant accepted a statement, that response was scored as one, and the rejection of a statement was scored as zero. The mean percentage acceptance by condition was computed by first converting a participant's score out of four (= \# of test trials) to a percentage, then arriving at the arithmetic mean of the twelve (= \# of participants per condition) percentage scores.

The overall results from the experiment are summarized in table (15). Our findings are: (i) regardless of negation type, speakers accept the $\forall>$ Neg reading; (ii) speakers accept the Neg $>\forall$ reading with $w a$-negation; (iii) only $54 \%$ of the speakers accept the $\mathrm{Neg}>\forall$ reading with regular negation.

Mean Percentage Acceptance
\begin{tabular}{|l||l|l|}
\hline Scope & Plain Negation & wa-Negation \\
\hline \hline$\forall>$ Neg & $98 \%$ & $98 \%$ \\
\hline Neg $>\forall$ & $54 \%$ & $94 \%$ \\
\hline
\end{tabular}

The results clearly show that the condition testing the Neg $>\forall$ reading with regular negation stands out in comparison to the other conditions. Unlike the other three conditions, the participants in this condition roughly show a 50-50 split in their responses, replicating the disagreement in the literature. The significance of the results in this condition is verified by a one-way ANOVA, comparing average scores across all four conditions. The end result is $[\mathrm{F}(3,44)=9.156, p<.0001]$, indicating an extremely high degree of statistical significance. Post-hoc analysis using the Tukey HSD test yields a significant $(p<.05)$ difference whenever the 'Neg $>\forall$, regular negation' condition is compared against one of the other three conditions. We take these results to indicate that our findings can be generalized out to the entire population of Japanese speakers.

\subsection{Discussion and Analysis}

In this section, we consider the two hypotheses in (8) against our findings and argue that Hypothesis 1 is superior.

According to our findings, almost all the speakers accepted the $\forall>$ Neg reading in the ' $\forall>$ Neg - regular negation' condition. This suggests that a structure is available in which NegP is hierarchically lower than the object QNP, as in (8a). However, a structure in which NegP is higher than the object QNP, as in (8b), is also consistent with the data. This is because the situation that meets the truth conditions 
of $\forall>$ Neg also meets the truth conditions of Neg $>\forall$. For instance, a situation in which Donald eats none of the oranges is also a situation in which Donald eats not all of the oranges. Hence, speakers could be accepting the statements in the ' $\forall>$ $\mathrm{Neg}$ - regular negation' condition with the Neg $>\forall$ generated by Hypothesis 2 . So, the data in this condition remains inconclusive in determining the superiority of the two hypotheses. This kind of problem is identified as 'the entailment problem' by Lidz and Musolino (2002). ${ }^{3}$

In the 'Neg $>\forall$ - regular negation' condition, only half of the speakers accepted the Neg $>\forall$ reading. This fact simply cannot be accounted for with Hypothesis 2, because this hypothesis can only generate the Neg $>\forall$ reading, hence incorrectly predicting $100 \%$ acceptance in this condition. With Hypothesis 1 in conjunction with verb raising, the split response in this condition can be accounted for. According to Hypothesis 1, NegP is c-commanded by the object QNP, accounting for the half of the speakers who rejected the Neg $>\forall$ reading. For the other half of the speakers, a structure must be available in which negation is higher than the object QNP. This can be obtained if the verb raises to $\mathrm{T}^{0}$, picking up negation on its way to $\mathrm{T}^{0}$, forming a complex head, as in (16). Assuming the Kaynean definition of c-command, as in (17), negation in $\mathrm{T}^{0}$ is able to c-command out of the complex head, and take scope over the object.

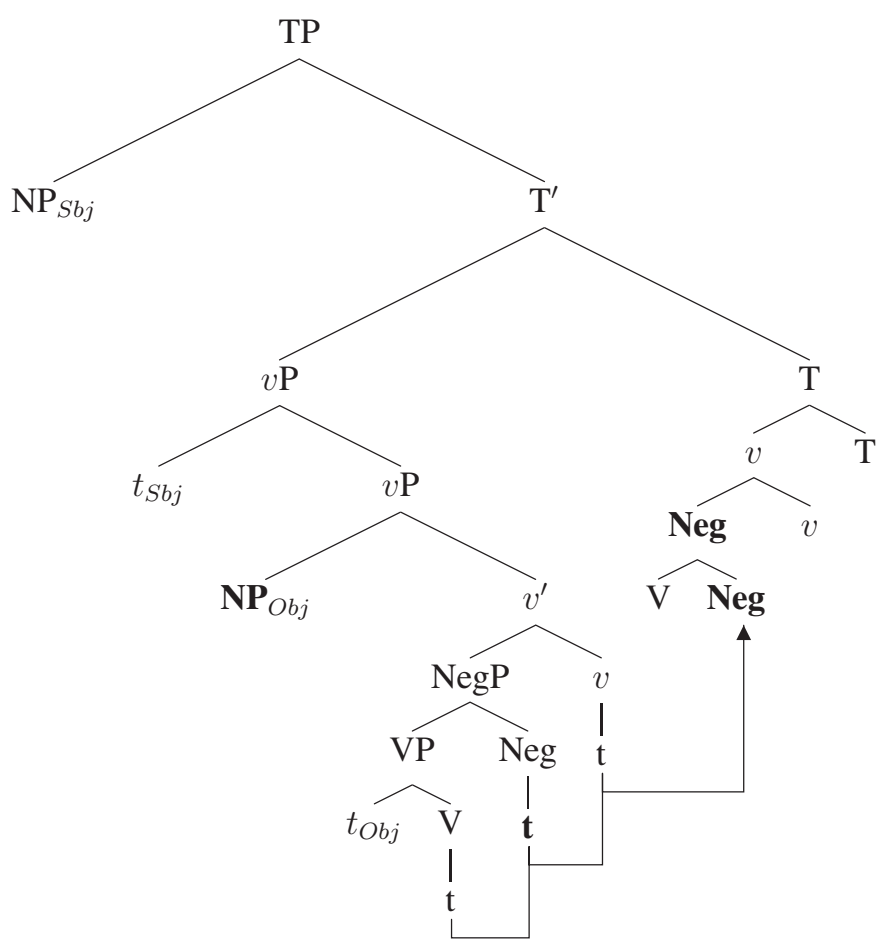

(17) X c-commands $\mathrm{Y}$ iff $\mathrm{X}$ and $\mathrm{Y}$ are categories and $\mathrm{X}$ excludes $\mathrm{Y}$ [no segment of $\mathrm{X}$ dominates $\mathrm{Y}$ ] and every category that dominates $\mathrm{X}$ dominates $\mathrm{Y}$ (Kayne, 1994, p. 16).

\footnotetext{
${ }^{3} \mathrm{~A}$ way to avoid the entailment problem and to obtain more conclusive data in this condition would be to use scenarios and test statements with numeral quantifiers. For example, in a scenario where Donald is given four oranges but eats only two of them, Donald did not eat two oranges is true under the Two $>$ Neg reading but false under the Neg $>$ Two reading. We are currently in the process of designing a new experiment with numerals.
} 
But our reasoning so far implies that only half of the speakers have verb raising. If all the speakers had verb raising, then all the speakers would incorrectly be predicted to accept the Neg $>\forall$ reading, just as predicted by Hypothesis 2 .

The idea that there may be variation concerning verb raising within a single speech community is not new. Han et al. (2003) report a similar TVJT experiment on scope of negation and object QNP in Korean with a similar split in scope judgments. Han et al. argue that this split is a by-product of the head-final clausal structure which obliterates string order evidence for verb raising. Thus, as far as verb raising is concerned, they argue, the learners of Korean do not receive enough evidence to decide the matter, and hence not all speakers acquire the same grammar. We take our results from Japanese, another head-final language, to be a further support of the proposal put forth in Han et al. and conclude that the Neg $>\forall$ reading with regular negation is available only to the speakers that have acquired a verb raising grammar. This proposal is further supported by the fact that the split in scope judgments is not within a speaker but between speakers: that is, a speaker either uniformly accepted $\mathrm{Neg}>\forall$ or uniformly rejected $\mathrm{Neg}>\forall$, and s/he did not accept or reject Neg $>\forall$ half of the time. ${ }^{4}$

We now need to recast the data in the ' $\forall>$ Neg - regular negation' condition in light of the split in verb raising. For the speakers who do not have verb raising, Hypothesis 1 straightforwardly generates the $\forall>$ Neg reading. For the speakers who have verb raising, negation will end up taking scope over the object QNP, generating the Neg $>\forall$ reading. But as discussed earlier, even these speakers will accept the statements given because the scenario that verifies $\forall>$ Neg also verifies Neg $>\forall$. This then accounts for the near $100 \%$ acceptance rate in this condition. ${ }^{5}$

Almost all speakers accepted the Neg $>\forall$ reading in the 'Neg $>\forall-w a$ negation' condition. What this means is that a structure is available for all speakers in which negation is higher than the object QNP. How could this be if negation is projected lower in the clause, as we just concluded? First of all, a clause structure containing wa-negation which is consistent with Hypothesis 1 is as in (18), where - $w a$ on the verb blocks the verb from coming together with the tense inflection, and instead suru 'do' is inserted in $\mathrm{T}^{0}$ to support tense. Further, exploiting the fact that $-n a$ has an affixal status morphologically, the Neg $>\forall$ reading can be generated if $n a$ moves and cliticizes onto suru in $\mathrm{T}^{0}$. We assume that $-n a$ adjoins to $\mathrm{T}^{0}$ in syntax and then it undergoes a morpho-phonological relinearization in morphology (in the sense of Embick and Noyer 2001 and Fukui and Sakai 2003) obtaining the surface string order suru+na+TENSE.

\footnotetext{
${ }^{4}$ Interestingly, the literature on Japanese does not seem to agree on whether Japanese has verb raising, just as there isn't much agreement on scope judgments concerning negation and quantified arguments. For instance, while Otani and Whitman (1991) and Koizumi (2000) argue for verb raising, Hoji (1998) points out a flaw in Otani and Whitman's argument, essentially concluding that their argument is not sufficient to support verb raising, and Fukui and Sakai (2003) refute all the arguments presented by Koizumi and argue for the position that Japanese has no verb raising. See Storoshenko (2004) for a summary of the literature on this issue. A similar state of affairs for Korean is reported and summarized in Han et al. (2003).

${ }^{5}$ If we were to use numerals instead of a universal quantifier in the test sentences, we would predict a split in responses in this condition as well. This prediction remains to be tested.
} 
(18)

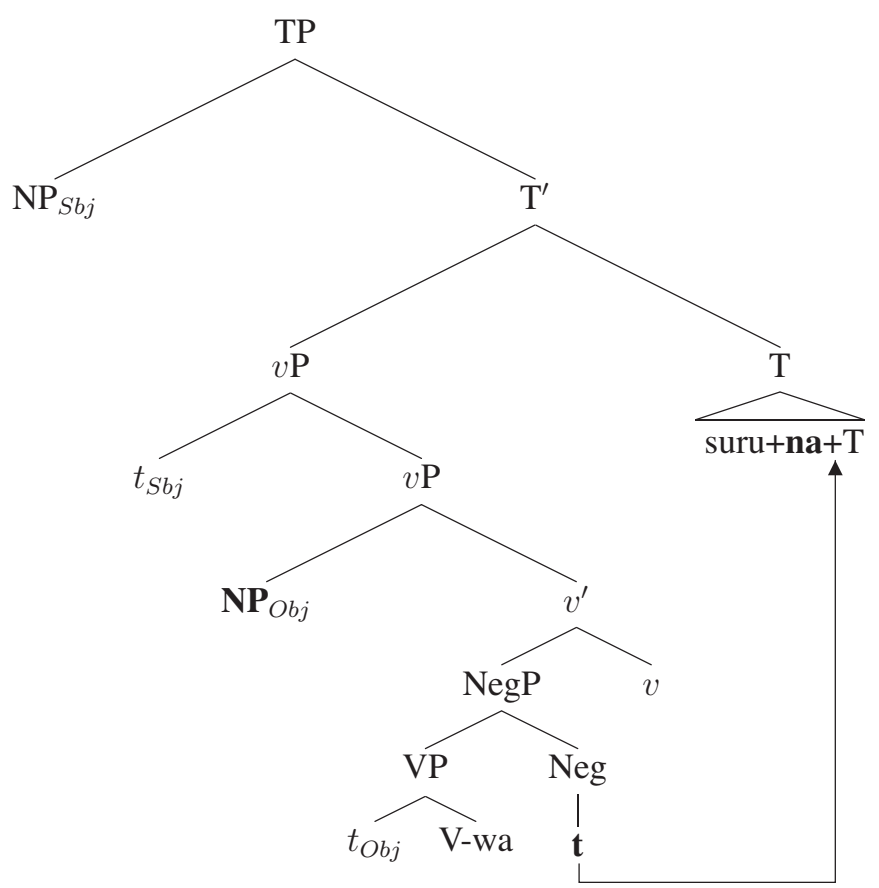

In the ' $\forall>$ Neg - wa-negation' condition, almost all speakers also accepted the $\forall>$ Neg reading. The structure in (18) is consistent with this finding. The $100 \%$ acceptance rate is expected because a context that verifies the $\forall>$ Neg reading also verifies the $\mathrm{Neg}>\forall$ reading. $^{6}$

\section{Conclusion}

In this paper, we have shown, through scope judgments obtained from a TVJT experiment, that Japanese speakers seem to uniformly accept the $\forall>$ Neg reading with regular and $w a$-negation, and the Neg $>\forall$ reading with $w a$-negation, but that there is a split in the population when it comes to the Neg $>\forall$ reading with regular negation. We have attributed this split in scope judgments to a split in the availability of verb raising within the population, and argued that all the data can be accounted for if negation is projected lower in the clause structure. With our analysis, we are able to present a unified account of the two types of sentential negation in Japanese, and also provide an explanation for the disagreement in the extant literature on the topic of negation scope as a reflection of speakers' grammars.

\section{References}

Chomsky, Noam. 1995. The Minimalist Program. Cambridge, MA: The MIT Press. Crain, Stephen, and Cecile McKee. 1985. The acquisition of structural restrictions on anaphora. In Proceedings of NELS 16. GLSA.

Crain, Stephen, and Rosaline Thornton. 1998. Investigations in Universal Grammar: A Guide to Research in Acquisition of Syntax and Semantics. Cambridge, Massachusetts: The MIT Press.

Embick, David, and Rolf Noyer. 2001. Movement operations after syntax. Linguistic Inquiry 32:555-595.

\footnotetext{
${ }^{6}$ With test sentences containing numerals, we predict a near $0 \%$ acceptance rate in this condition.
} 
Fukui, Naoki, and Hiromu Sakai. 2003. The visibility guideline for functional categories: Verb raising in Japanese and related issues. Lingua 113:321-375.

Han, Chung-hye, Jeffrey Lidz, and Julien Musolino. 2003. Verb-raising and grammar competition in Korean: Evidence from negation and quantifier scope. Manuscript, Simon Fraser University, Northwestern University, Indiana University.

Hoji, Hajime. 1985. Logical form constraints and configurational structure in Japanese. Doctoral Dissertation, University of Washington.

Hoji, Hajime. 1998. Null object and sloppy identity in Japanese. Linguistic Inquiry 29:127-152.

Kayne, Richard. 1994. The Antisymmetry of Syntax, volume 25 of Linguistic Inquiry Monograph. Cambridge, London: The MIT Press.

Koizumi, Masatoshi. 1995. Phrase structure in minimalist syntax. Doctoral Dissertation, MIT.

Koizumi, Masatoshi. 2000. String vacuous overt verb raising. Journal of East Asian Linguistics 9:227-285.

Kuno, Susumu. 1973. The Structure of the Japanese Language. Cambridge, MA: MIT Press.

Kuno, Susumu. 1980. The scope of question and negation in some verb-final languages. In Proceedings of the Chicago Linguistic Society, ed. Jody Kreiman and Almerindo E. Ojeda, volume 16, 155-169. Chicago Linguistic Society.

Kuroda, Shige-Yuki. 1979. Generative Grammatical Studies in Japanese Language. New York, London: Garland Publishing.

Lidz, Jeffrey, and Julien Musolino. 2002. Children's command of quantification. Cognition 84:113-154.

Miyagawa, Shigeru. 2001. The EPP, scrambling and wh-in-situ. In Ken Hale: A Life in Language, 293-338. Cambridge, MA: MIT Press.

Miyagawa, Shigeru. 2002. The nature of weak islands. Conference handout, KGGC Summer School.

Ota, Akira, and Yasuhiko Kato. 1986. The relative scope of neg and quantifiers in English and Japanese. Sophia Linguistica 20-21:25-40.

Otani, Kazuyo, and John Whitman. 1991. V-raising and VP-ellipsis. Linguistic Inquiry 22:345-358.

Rizzi, Luigi. 1990. Relativized Minimality. Cambridge, MA: MIT Press.

Storoshenko, Dennis Ryan. 2004. Negation scope and phrase structure in Japanese. Master's thesis, Simon Fraser University.

Yatabe, Shuichi. 1996. Negation and focusing in the grammar of Japanese. In Studies on the Universality of Constraint-based Phrase Structure Grammars, ed. Takao Gunji, 217-225. Osaka, Japan: Osaka University.

Chung-hye Han, Dennis Ryan Storoshenko, Yasuko Sakurai

8888 University Drive

Department of Linguistics

Simon Fraser University

Burnaby, BC V5A1S6, Canada

\{chunghye,dstorosh,ysakurai\}@sfu.ca 